\title{
Beneficios tributarios como soporte de competitividad y emprendimiento para MiPymes
}

Nancy María Sánchez R. *

Sánchez R., N.M. (2011). Beneficios tributarios como soporte de competitividad y emprendimiento para MiPymes. Revista Activos, 16 y 17, 17-31.

JEL: H25

Recibido: 2 de marzo de 2011 Aprobado: 28 de marzo de 2011

\section{Resumen}

Dada la evolución que en Colombia ha tenido la economía y la necesidad de estimular los negocios y la generación de empleo, los gobiernos tanto nacionales como regionales han establecido como estrategias de desarrollo unos estímulos que benefician la creación de empresas, dentro de los cuales se mencionan los de carácter tributario, que revisten de gran importancia ya que a la hora de emprender un negocio no se puede olvidar el impacto que los tributos tienen en el interior del giro ordinario de las compañías.

* Contadora Pública, profesora de tiempo completo de la Facultad de Contaduría Pública de la Universidad Santo Tomás, Villavicencio. 


\title{
Palabras clave
}

Beneficio, competitividad, descuentos, emprendimiento, exenciones, parafiscales, progresividad, soporte, tarifas, tributario.

Sánchez R., N.M. (2011). Tax Aids as a Support for Competivity and Venture in SMEs. Activos Review, 16 and 17, 17-31.

\begin{abstract}
Given the evolution of the Colombian economy and the need to stimulate the business and job creation, the national and local government have established, as development strategies, encouragements that benefit the enterprise creation. Among them we can count tax stimulus, that have great importance, because at the moment of beginning with a business you cannot forget the impact of taxes inside of the companies.
\end{abstract}

\section{Keywords}

Aid, competivity, discounts, venture, exemption, parafiscal, progress, support, tariff, tributary.

Sánchez R., N.M. (2011). Bénéfices tributaires comme support de compétitivité et d'esprit d'entreprise pour les MIPMES. Revue Activos, 16 et 17, 17-31.

\section{Résumé}

Vu l'évolution de l'économie en Colombie et la nécessité de stimuler les affaires et la création d'emplois, tant les gouvernements nationaux que régionaux ont établi comme stratégies de développement des stimuli qui bénéficient à la création d'entreprise, parmi lesquels se distinguent ceux à caractère tributaire, qui revêtent une grande importance, puisqu'au moment de se lancer dans une entreprise, on ne peut pas oublier l'impact que les impôts ont à l'intérieur de la conduite habituelle des affaires. 


\section{Mots-clés}

Bénéfice, compétitivité, décomptes, esprit d’entreprise, exemptions, parafiscal, progressivité, support, tarifs, tributaire.

\section{Introducción}

Hace algún tiempo crear empresa era un sueño utópico o sólo de muy pocos que manejaran poder, dinero y estudio. Ahora este sueño reviste cada vez más realidad, gracias a la evolución de los mercados -que son promotores y transformadores de las naciones y de la sociedad en general en este mundo globalizado- y al apoyo que encuentran por parte de los medios de comunicación. Las pequeñas compañías están constituidas, en su gran mayoría, por miembros de una familia que se agrupan con el fin de generar recursos para el sostenimiento de la misma o por jóvenes estudiantes en busca de mayores oportunidades, con miras a crecer de manera independiente inducidos por la falta de oportunidades laborales. Desafortunadamente, estas pequeñas empresas, al igual que los emprendedores informales, conocen de manera superficial o, en el peor de los casos, no conocen los apoyos que tienen como soporte para la creación de empresas; circunstancia que genera resistencia y miedo a la hora de emprender en un mundo que para ellos es desconocido.

Es por esta razón que se elabora el presente artículo, que ha de ser utilizado por este tipo de empresarios-contribuyentes como herramienta fundamental a la hora de planear y constituir su negocio en este país, el cual adolece de una economía estable; para ello necesariamente habrá que tener en cuenta el tema de los impuestos, dadas las constantes reformas tributarias.

El artículo está estructurado de tal manera que muestre la gama de beneficios tributarios ofrecidos tanto en el ámbito nacional como en el regional. Específicamente se enfoca en los departamentos del Meta y Casanare y, dentro de estos, en la ciudad de Villavicencio y sus municipios colindantes (Acacías, Cumaral, Restrepo y Granada), y en la ciudad de Yopal, respectivamente. Los beneficios a los que se hace 
alusión son aplicables a los aportes parafiscales ${ }^{1}$, al impuesto de renta ${ }^{2}$ y al impuesto del ICA ${ }^{3}$, en aquellas empresas cuyas actividades se desarrollan en los sectores de servicios, comercio e industria. Para esto se diseñó un normograma que ilustra de manera clara el efecto de los mismos.

Es importante apuntar que a pesar de que se habla solamente de los departamentos de Meta y Casanare, no se puede dejar de lado los beneficios que a nivel nacional existen, ya que estos también aplican para los otros departamentos.

\section{Los esfuerzos de los gobiernos nacional y regional}

El gobierno, a fin de no ser indiferente y de cara a una sociedad contemporánea que contribuya con el desarrollo del país, debe incluir dentro de su plan de acción políticas y estrategias que estimulen el emprendimiento, fomenten la internacionalización de los mercados y posibiliten, en gran medida, el proceso de desarrollo y mejoramiento de la calidad de vida de sus ciudadanos.

Se atribuye singular importancia a este apoyo, toda vez que en un mundo de mercados internacionales, la definición y establecimiento de procesos, compromisos y actividades -los cuales han de desbordar el ámbito nacional y considerar vínculos con otras instituciones locales, regionales o nacionales- cobran cada vez mayor fuerza y se constituyen en un requisito fundamental en términos de crecimiento y desarrollo. Para tal efecto, el gobierno ha venido desarrollando diferentes actividades encaminadas a potenciar la participación de la comunidad en las diferentes regiones y a abrir espacios nacionales y territoriales, mediante la unión de sinergias entre las colectividades.

En la orinoquía colombiana (departamentos del Meta y Casanare y en especial los municipios colindantes de Villavicencio y Yopal) desde la década de los noventa se ha presentado un crecimiento económico, el cual va de la mano de la inversión e inyección de capital para todos los sectores, con énfasis en el gran aporte que hace

1 Incluye los aportes para cajas de compensación familiar, Instituto Colombiano de Bienestar Familiar y el Sena.

2 Incluye el Impuesto de Renta y sus complementarios.

3 Impuesto de Industria y Comercio, Avisos y Tableros. 
el turismo al sector comercial y de servicios. Además, este desarrollo ha sido posible por la participación del Estado, el cual aporta en el desarrollo de una infraestructura que contribuye a la generación de nuevas empresas, sobre todo en el nivel de micro, pequeña y mediana. Por su parte, en los sectores de industria, comercio y servicios, el buen estado de las vías y el fácil acceso y salida en cada uno de los municipios ha contribuido con el mencionado crecimiento económico.

Por las razones anteriormente expuestas, se realizaron una serie de consultas con el propósito de ilustrar claramente los beneficios tributarios en estos municipios, tanto en impuestos nacionales de renta y complementarios como en los territoriales de industria y comercio. Mediante un cuadro comparativo se muestra cómo estos beneficios se ven reflejados en las empresas de la región. Es importante señalar que se tiene como punto de referencia a Bogotá, ya que su participación en los sectores mencionados presenta un gran desarrollo no sólo como ciudad capital, sino como líder en la creación de nuevas empresas, al estimular el emprendimiento y, por ende, la generación de nuevos empleos.

\section{Beneficios tributarios}

El gobierno de Colombia, mediante el Congreso de la República, a partir de la promulgación de la Ley 488 de 1998 otorgó descuentos tributarios en la declaración de renta de las empresas por los nuevos empleos directos que generaran. Así mismo, desde la promulgación de la Ley 590 de 2000, reglamentada por el artículo 43 del Decreto 525 de 2009 y de la Ley 905 de 2004, concedió una reducción en los aportes parafiscales de las MiPymes ${ }^{4}$. Igualmente, se encuentra la más reciente reforma dada en la Ley 1429 de 2010, que en general amplía estos beneficios tributarios para las pequeñas empresas ${ }^{5}$, cuyos artículos 5, 7, 8 y 48 están reglamentados por el Decreto 545 de 2011.

Señala la Ley las siguientes condiciones: Microempresas: deben tener máximo 10 empleados y el valor de sus activos debe ser inferior a 500 SMMLV, para 2011 \$ 267.800.000. Pequeña empresa: debe tener entre 11 y 50 empleados y el valor de sus activos debe estar entre 501 y 5000 SMMLV, es decir, entre \$268.335.600 y \$2.678.000.000. La mediana empresa: debe tener entre 51 y 200 empleados y el valor de sus activos debe estar entre 5001 y 30.000 SMMLV, es decir, entre \$2.678.535.000 y \$ 16.068.000.00. Por su parte, el parágrafo 1 del artículo 2 de la Ley 590 establece que el factor determinante para dicho efecto será el de activos totales.

El concepto de pequeñas empresas reúne a las microempresas y pequeñas empresas. 
Estos beneficios tributarios han sido creados como estrategia para estimular e incentivar la generación de empleo, contribuir con la competitividad y el emprendimiento y favorecer la creación y sostenibilidad de las empresas formales a nivel nacional. Con este mismo propósito, las autoridades de las entidades territoriales, desde la autonomía que se les confiere en la Constitución Política, fijan tarifas favorables en sus tributos locales. Los planteamientos propuestos anteriormente encuentran su razón de ser a través de las respuestas a los siguientes interrogantes: ¿qué beneficios tributarios estimulan la competitividad y el emprendimiento en el ámbito Nacional? ¿Cómo estos beneficios se ven reflejados en los departamentos de Meta y Casanare?

Como resultado de estas consultas se encuentra que los beneficios fiscales se clasifican en beneficios de orden nacional y de orden territorial, aunque estos últimos son específicamente municipales, dado que a nivel departamental no es posible vincularlos, pues no hay un impuesto departamental general aplicable a los sectores de comercio, industria y servicios, como en el caso del ICA. Los impuestos departamentales tocan sectores específicos como el consumo de cerveza, cigarrillos, licores, gasolina, registro, etc., y a estos sectores no apuntan los emprendedores de MiPymes como oportunidades de negocio. Los beneficios de orden nacional son de dos tipos: beneficios en el impuesto de renta y complementarios (en los que se encuentran tarifas progresivas y descuentos tributarios) y reducción en los aportes parafiscales, con tarifas progresivas también.

A continuación se presenta un normograma en el que se relacionan las normas que reglamentan los impuestos nacionales y refieren los beneficios señalados. El cuadro permite una visión clara y esquematizada y da cuenta del trabajo realizado, a fin de satisfacer las necesidades que tienen los contribuyentes de conocer la regulación a la que pueden acogerse a la hora de emprender y formalizar empresa en el país, más exactamente en los departamentos del Meta y Casanare, con énfasis en los municipios colindantes de Villavicencio y Yopal. 
Beneficios tributarios como soporte de competitividad y emprendimiento para MiPymes | ACTIVOS

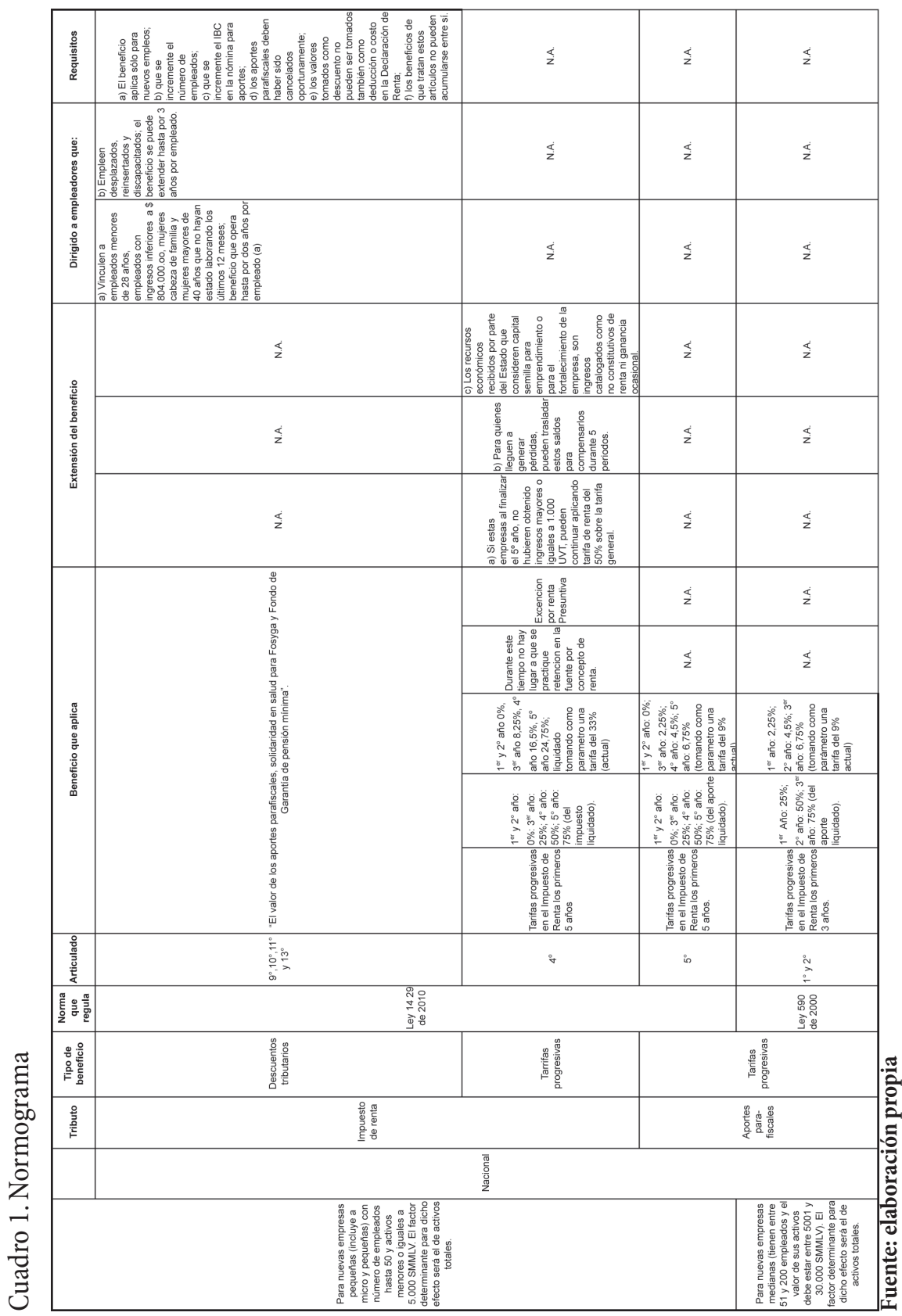


En general, los beneficios de orden nacional se destacan en el impuesto de renta y complementarios, en la sección de liquidación privada del formulario 110 para el año 2010 y siguientes, al liquidar el impuesto con base a la tarifa. Así mismo, sobresalen los descuentos que se reportan en el renglón 70 del formulario, los cuales restan al impuesto sobre la renta líquida gravable previamente liquidado, generando así el impuesto neto de renta a reportar en el renglón 71 y el tiempo estipulado, ya que el mismo beneficio se puede extender hasta por dos años.

En los beneficios de orden territorial pueden mencionarse como exenciones en el impuesto de industria y comercio las tarifas especiales y descuentos. Estos se encuentran respaldados en el artículo 42 de la Ley 590 de 2000, la cual faculta a los municipios, distritos y departamentos para establecer regímenes especiales sobre impuestos, tasas y contribuciones de orden territorial, con el fin de estimular la creación y subsistencia de las MiPymes, para lo cual podrán establecer exclusiones, períodos de exoneración y tarifas inferiores a las ordinarias.

Así mismo, el artículo 6 de la Ley 1429 de 2010 exhorta a las entidades territoriales del país para que de manera voluntaria apliquen en el impuesto de industria y comercio el mismo principio de progresividad, de tal forma que éste opere de la misma manera que el impuesto de renta y así se articulen los aportes parafiscales.

En la ciudad de Villavicencio, a través del el artículo 61 del Acuerdo Municipal 030 de 2008, en el aparte de incentivos fiscales, se establece un descuento en el impuesto de industria y comercio, incluyendo el impuesto de avisos y tableros, del 8\%, si el pago se hace simultáneamente con la declaración y como mínimo con un mes de antelación a la fecha de vencimiento.

Por su parte, el artículo 95 del Acuerdo 044 de 2008, referente a los incentivos fiscales, establece para el municipio de Restrepo:

Los Contribuyentes del Impuesto de Industria y Comercio, Avisos y Tableros tendrán derecho al siguiente incentivo fiscal: quienes presenten la declaración y paguen el Impuesto de manera oportuna tendrán derecho a un descuento del cuatro por ciento (4\%), como beneficio fiscal, liquidado sobre el impuesto incluyendo el de avisos y tableros. 
Mientras que en Cumaral, en el marco fiscal del mediano plazo 2008-2011, numeral 6 , se aclara que durante este período no hay beneficios fiscales en el municipio que tengan relación con el impuesto de industria y comercio.

Por su parte, en Granada, el estatuto de rentas municipales (artículo 67 del Acuerdo 037 del año 2004 del Concejo Municipal) exonera del hecho generador a las profesiones liberales, por cuanto quedan exentas del impuesto de industria y comercio. De igual modo establece un descuento del 10\%, tomando como base el impuesto de industria y comercio generado, en el caso de cumplirse oportuna y simultáneamente con la obligación formal ${ }^{6} \mathrm{y}$ con la obligación sustantiva ${ }^{7}$.

Se destaca que en Acacías, en el departamento del Meta, los beneficios que apoyan el emprendimiento y la competitividad son interesantes para quien desee establecer empresa allí, toda vez que a la luz del Acuerdo Municipal No. 048 de 2008 se están otorgando beneficios tributarios en el "impuesto de industria y comercio para apoyar el emprendimiento y generación de empleo". Específicamente en el artículo 66 de dicho acuerdo se establece:

Las empresas que se creen, constituyan o establezcan en el municipio de Acacías y que generen empleos o como mínimo el 70\% del personal contratado, sea oriundo del municipio de Acacías o que tenga mínimo cinco años de residencia, previa certificación de la Secretaría de Gobierno y presentación del certificado electoral, tendrán una exención en el impuesto de industria y comercio teniendo en cuenta las siguientes condiciones:

Empresas que generen:

- Mínimo el 20\% del personal en diversas funciones y que sean oriundos de Acacías tendrán un descuento del 20\% del mencionado impuesto.

- Entre 4 y 9 empleos quedarán exentos del $35 \%$ del anterior impuesto.

- Entre 10 y 19 empleos quedarán exentos del 50\% del impuesto.

6 Obligación de informar y "presentar la declaración".

7 Obligación de dar por parte del sujeto pasivo y de recibir por parte del sujeto activo. 
- Entre 20 y 29 empleos quedarán exentos del $80 \%$ y

- De 30 empleos en adelante quedarán exentos del 100\% del ICA.

La base que se tiene en cuenta para liquidar esta exención es el impuesto que se determine en el período gravable en el cual se acoja a la exención.

En Yopal, el estatuto de rentas municipales establece un descuento del 10\%, tomando como base el impuesto de industria y comercio generado, en el caso de cumplirse oportunamente con la declaración y su correspondiente pago. Este descuento aplica únicamente para los contribuyentes del régimen simplificado.

Gráfica 1. Tarifas para los sectores de servicios, industria y comercio en el ICA

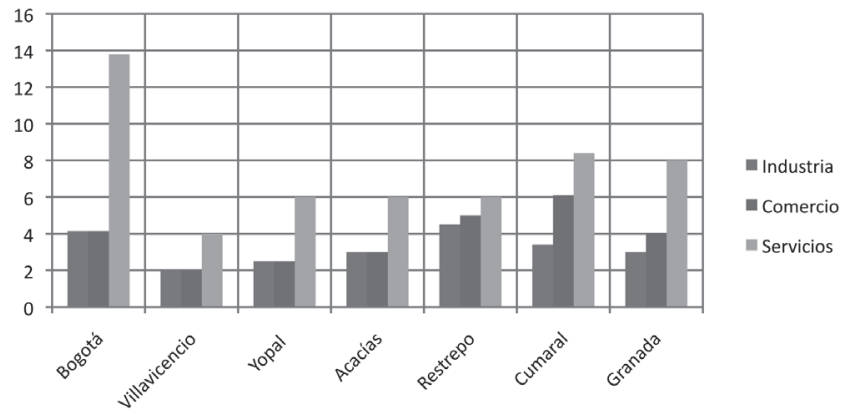

\section{Fuente: elaboración propia}

En el anterior gráfico se establece un punto de comparación de las tarifas diferenciales que operan en cada uno de los municipios, tomando como punto de referencia la ciudad de Bogotá, ya que esta es líder en emprendimiento y competitividad a nivel nacional. Como se puede observar, las tarifas del impuesto de industria y comercio en la región son muy favorables, lo que hace más atractiva la creación de empresa y generación de empleo.

Las tarifas que se tienen en cuenta como punto de referencia en el gráfico anterior son: para el sector de servicios aplica la de hoteles y alojamiento, para el sector comercial aplica la de venta de alimentos, para el sector industrial aplica la tarifa de elaboración de productos alimenticios.

La gráfica muestra que el sector de servicios es el que tiene tarifas más altas; sin embargo, en Villavicencio están las tarifas más bajas, seguido de Yopal, Acacías y 
Restrepo. Por su parte, Cumaral y Granada, aunque parecen tener las tarifas más altas de la muestra, no alcanzan a llegar al nivel de la ciudad de Bogotá, lo que permite concluir que en la región vale la pena invertir en el sector de los servicios.

En cuanto a los sectores de industria y comercio, estos son los más atractivos por sus tarifas. En Villavicencio y Yopal son muy similares; en Acacías y Restrepo, aunque las tarifas se incrementan, los dos sectores son similares; en Cumaral y Granada el sector comercio está gravado en un nivel más alto que el industrial; sin embargo, es importante destacar que en los tres sectores las tarifas son más favorables que en Bogotá.

Vale acotar que se toman tarifas en condiciones normales, ya que en Acacías, como se dijo con anterioridad, si hay generación de empleo, las empresas quedan excluidas del impuesto de manera proporcional.

Complementado lo anterior, en la siguiente gráfica se presenta una simulación sobre el valor a pagar por impuesto de industria y comercio en los municipios que forman parte de la muestra. Se toma como referencia una base gravable de \$50.000.000 para las empresas cuyas actividades económicas y tarifas fueron las ilustradas en el gráfico 1.

Gráfica 2. Ejemplo de impuesto de industria y comercio

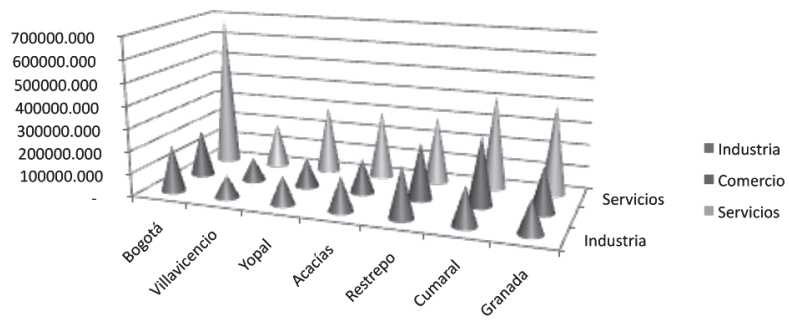

Fuente: elaboración propia.

Los resultados permiten evidenciar que el sector de servicios de alojamiento en la ciudad de Bogotá pagaría aproximadamente $\$ 700.000$ (setecientos mil pesos) por impuesto de industria y comercio, mientras que en la ciudad de Villavicencio, por el mismo servicio y en iguales condiciones, se presenta un impuesto de $\$ 250.000$ (doscientos cincuenta mil pesos), lo que constituye una diferencia bastante marcada. Ahora bien, en el sector de comercio de alimentos el impuesto en Bogotá supera los \$100.000 (cien mil pesos), mientras que en Villavicencio es inferior, como ya se 
indicó en las mismas condiciones de base y actividad.Y para el sector de industria de alimentos, en Bogotá opera un impuesto que también supera los $\$ 100.000$, mientras que en Villavicencio está por debajo de esta cifra. Sin embargo, los municipios de Restrepo y Cumaral tienen un impuesto más alto que en los otros municipios de la región. El procedimiento que deben seguir los contribuyentes para hacer uso del beneficio es el que se enumera a continuación:

1. En el registro mercantil, informar a las cámaras de comercio, mediante el formulario de inscripción o, en su defecto, mediante declaración juramentada, el hecho de cumplir condición de pequeña empresa.

2. Radicar ante la $\mathrm{DIAN}^{8}$ una carta con tres copias que indique la intención de acceder a los incentivos parafiscales. La DIAN suministrará un código en el RUT, con el fin de que puedan liquidar los aportes disminuidos en la planilla unificada de pagos. Ésta suministrará al Ministerio de la Protección Social la información relacionada con la empresa que se acoge al beneficio.

Es de aclarar que el primer año de operación de la empresa se contará a partir de la fecha reportada como inicio de la actividad económica principal en el Registro Mercantil.

Así mismo, estas empresas gozan de unos beneficios, que aunque no son fiscales, estimulan el emprendimiento, como es el caso del pago de Registro Mercantil y su renovación, a saber:

- Para el Registro Mercantil se pagará el 0\% del valor liquidado.

- Para renovación del Registro Mercantil en el segundo año de formalización se pagará el 50\% del valor liquidado.

- Para renovación del Registro Mercantil en el tercer año de formalización se pagara el 75\% del valor liquidado.

\section{Conclusiones}

De acuerdo a lo anteriormente mencionado, es necesario recalcar varios aspectos que le han permitido a la región una evolución notable en cuanto a su desarrollo económico. Especialmente es importante señalar los beneficios que esto tiene en 
relación con una mejora en su infraestructura y una tasa de paro favorable, que gracias a los beneficios tributarios y a otros que el Estado brinda por la generación de nuevas empresas, y que fueron ilustrados a lo largo de este documento, han hecho que la región tenga un crecimiento y desarrollo importante en cuanto a la competitividad y el emprendimiento de sus empresas.

En este sentido, el interés que ha traído consigo la sociedad por crear empresas y los beneficios que presta el gobierno de la región han favorecido su crecimiento, el cual se ve representado en el número de emprendedores, quienes gracias a su interés por salir adelante acogen dichos beneficios y emprenden de manera notable iniciativas de este tipo. Con esto se ayuda a la generación de empleo y se genera una evolución significativa en la infraestructura y una importante atracción hacia la inversión nacional y extranjera en la región.

Dadas las constantes reformas tributarias, es vital tener muy presentes los impuestos para la creación de una nueva empresa. La alta gama de beneficios tributarios ofrecidos a nivel nacional y la atracción que generan las demás ventajas de la región logra incentivar el desarrollo de la misma. En este panorama, resultan muy significativas las estrategias utilizadas por el gobierno con el fin de estimular el emprendimiento y fomentar el mejoramiento de la región en general y de la calidad de vida de cada uno de sus ciudadanos.

El importante desarrollo económico que ha presentado la región la hace atractiva a la inversión e inyección de capital en cada uno de sus sectores. A esto se une la captación de turismo que sostiene el comercio de bienes y servicios, además de un excelente desarrollo en su infraestructura y las importantes vías de acceso y salida, que permiten la entrada a nuevos mercados nacionales e internacionales.

Finalmente, es necesario resaltar aspectos como la salud y la educación. La inversión que se hace en ellos en la región mejora significativamente la calidad de vida de cada uno de sus habitantes; la importancia de la educación se ve reflejada en la cantidad de jóvenes profesionales que tienen el conocimiento y las ganas de salir adelante. De la mano de las políticas y estrategias que el Estado brinda, la educación fomenta el crecimiento y desarrollo económico de la región, hace posible la creación de nuevas empresas y estimula el crecimiento individual y colectivo. 


\section{Referencias}

Congreso de la República de Colombia (1998). Ley 488 de 24 de diciembre de 1998, por la cual se expiden normas en materia tributaria y se dictan otras disposiciones fiscales de las Entidades Territoriales. Diario Oficial, núm. 43.460, 28 de diciembre de 1998. Recuperado de http://www.minhacienda.gov.co/ MinHacienda/haciendapublica/regulacionfinancieraysocial/RegistroUnicodeAportantes/normatividad

Congreso de la República de Colombia (2000). Ley 590 de 10 de julio de 2000, por la cual se dictan disposiciones para promover el desarrollo de las micro, pequeñas y medianas empresas. Diario Oficial, núm. 444.078, 12 de julio de 2000. Recuperado de http://www.secretariasenado.gov.co/senado/basedoc/ ley/2000/ley_0590_2000.html

Congreso de la República de Colombia (2004). Ley 905 de 2 de agosto de 2004, por medio de la cual se modifica la Ley 590 de 2000 sobre promoción del desarrollo de la micro, pequeña y mediana empresa colombiana y se dictan otras disposiciones. Diario Oficial, num.45.628, 2 de agosto de 2004. Recuperado de http:// www.secretariasenado.gov.co/senado/basedoc/ley/2004/ley_0905_2004.html

Congreso de la República de Colombia (2010). Ley 1429 de 29 de diciembre de 2010, por la cual se expide la Ley de Formalización y Generación de Empleo. Diario Oficial, núm. 47.937, 29 de diciembre de 2010. Recuperado de http:// www.secretariasenado.gov.co/senado/basedoc/ley/2010/ley_1429_2010.html

Ministerio de Comercio, Industria y Turismo de Colombia (2009). Decreto 525 de 23 de febrero de 2009, por medio del cual se reglamenta el artículo 43 de la Ley 590 de 2000 y demás normas concordantes. Recuperado de http://camara.ccb. org.co/documentos/4475_decreto525de2009.pdf

Ministerio de la Protección Social de Colombia (2011). Decreto 545 de 25 de febrero de 2011, por el cual se reglamentan parcialmente los artículos 5, 7, 48 y 50 de la Ley 1429 de 2010. Recuperado de http://camara.ccb.org.co/documentos/7805_Decreto545_25Feb2011.pdf 
Municipio de Acacías (2008). Acuerdo 048 de 2008 del Honorable Concejo Municipal. Recuperado de http://www.Acacias-meta.gov.co/index.shtml

Municipio de Cumaral (2008). Marco Fiscal del Mediano Plazo 20082011. Recuperado de http://www.cumaral-meta.gov.co/apc-aa-files/66313164656438653138616435636330/MFMP_CUMARAL_2008.pdf

Municipio de Granada (2001).Estatuto Tributario,Acuerdo 037 de 2001. Recuperado de http://www.Granada-meta.gov.co/index.shtml

Municipio de Restrepo (2008). Acuerdo 044 de 2008 del Honorable Concejo Municipal. Recuperado de http://www.Villavicencio-meta.gov.co/index.shtml

Municipio de Villavicencio (2008). Acuerdo 030 de 2008 del Honorable Concejo Municipal. Recuperado de http://www.Villavicencio-meta.gov.co/index.shtml

Municipio de Yopal (2004). Acuerdo 013 de 2004 del Honorable Concejo Municipal, por medio del cual se expide el "Código de Rentas para el Municipio de Yopal". Recuperado de http://www.yopal-casanare.gov.co/apc-aa-files/38336239393262616235333063343464/CODIGO_DE_RENTAS_MUNICIPAL.pdf 